\title{
Inspiratory training and immediate lung recovery after resective pulmonary surgery: a randomized clinical trial
}

\author{
Sabina Lähteenmäki ${ }^{1,2}$, Thanos Sioris ${ }^{2}$, Heidi Mahrberg ${ }^{2}$, Irina Rinta-Kiikka ${ }^{3}$, Jari Laurikka ${ }^{1,2}$ \\ ${ }^{1}$ Faculty of Medicine and Health Technology, Tampere University, Tampere, Finland; ${ }^{2}$ Tampere Heart Hospital, Tampere, Finland; ${ }^{3}$ Imaging Centre, \\ Department of Radiology, Tampere University Hospital, Tampere, Finland \\ Contributions: (I) Conception and design: J Laurikka, H Mahrberg, I Rinta-Kiikka; (II) Administrative support: J Laurikka, I Rinta-Kiikka, T Sioris; (III) \\ Provision of study materials or patients: All authors; (IV) Collection and assembly of data: J Laurikka, H Mahrberg, I Rinta-Kiikka, S Lähteenmäki; (V) \\ Data analysis and interpretation: All authors; (VI) Manuscript writing: All authors; (VII) Final approval of manuscript: All authors. \\ Correspondence to: Sabina Lähteenmäki. Korentijärventie 130, 37600 Valkeakoski, Finland. Email: sabina.lahteenmaki@tuni.fi.
}

Background: Prompt and uneventful recovery after resective pulmonary surgery benefits patients by decreasing length and total costs of hospital stay. Postoperative physiotherapy has been shown to be advantageous for patient recovery in several studies and lately inspiratory muscle training (IMT) physiotherapy has been used also in thoracic patients. This randomized controlled trial intended to evaluate whether IMT is an efficient and feasible method of physiotherapy compared to water bottle positive expiratory physiotherapy (PEP) immediately after lung resections.

Methods: Forty-two patients were randomly allocated into two intervention groups: water bottle PEP $(\mathrm{n}=20)$ and IMT group $(\mathrm{n}=22)$. Patients were given physiotherapeutic guidance once a day and patients were also instructed to do independent exercises. Measurements of pulmonary function were compared between the treatment groups according to intention to treat by using two-way repeated measures analysis of variances at three time points (preoperative, first postoperative day, and second postoperative day). Walking distance was measured at first and second postoperative day and similarly, evaluation of postoperative air leak during exercises was performed. Physiotherapy was modified or temporarily interrupted, if necessary, because of the air leak.

Results: Postoperative pulmonary function tests were equal between the intervention groups. Air leak was relatively common after lung resections: $31 \%$ of all patients had mild or moderate/severe air leak at first postoperative day and $14 \%$ of all patients had mild to severe air leak at second postoperative day respectively. There were no statistically significant differences in occurrence of air leak between intervention groups, but water resistance had to be reduced or physiotherapy discontinued significantly more often among the water bottle PEP group patients $(\mathrm{P}=0.01)$. Walking distance improved slightly faster in the IMT group between the first and the second postoperative day when compared to the water bottle PEP group, but the difference between the groups was not statistically significant.

Conclusions: IMT physiotherapy is equally effective to water bottle PEP training in postoperative physiotherapy after lung resection surgery evaluated with pulmonary function tests and walking distance. In addition, IMT physiotherapy is safe and more feasible form of physiotherapy during postoperative air leak compared to water bottle PEP.

Keywords: Pulmonary; postoperative complication; air leak; postoperative care

Submitted Apr 21, 2020. Accepted for publication Sep 18, 2020.

doi: $10.21037 /$ jtd-20-1668

View this article at: http://dx.doi.org/10.21037/jtd-20-1668

ㄷ Journal of Thoracic Disease. All rights reserved. 


\section{Introduction}

Lung resection patients benefit from prompt and uneventful recovery, which also reduces the duration and overall cost of hospitalization. Patient's postoperative recovery may be impaired due to wound pain, impaired diaphragmatic function or reduced volume of the lung and thereby reduction in ventilated air. Attenuated respiratory function may expose to development of atelectasis, mucus accumulation, pneumonia, and other postoperative pulmonary complications (PPC), especially in high risk patients (1-5).

Postoperative physiotherapy has been shown to be advantageous for patient recovery in several studies (3,6-19). Rehabilitation before and after lung cancer surgery is recommended also in the ERS/ESTS (European Respiratory Society/European Society of Thoracic Surgeons) rehabilitation guidelines for a lung cancer patient (19). Water bottle positive expiratory pressure (PEP), which is performed by blowing bubbles through a large-bore (ca.10 millimetre) tube into water filled bottle, is widely used for respiratory training after thoracic surgery. Lately also inspiratory muscle training (IMT) physiotherapy has been used in these patient groups. In IMT inhalation takes place against resistance, which is proportional to patient's own maximal inspiratory force. When combined with upper and lower limb exercises, IMT has shown statistically significant improvement in lung function tests and in six-minute walking tests (20). IMT physiotherapy has also reduced the incidence of PPC and duration of hospitalization with lung surgery patients $(12,21-23)$. In addition, IMT physiotherapy has increased the distances of six-minute walking test with lung transplantation patients (24) and improved exercise tolerance in COPD patients $(25,26)$.

Aim of this prospective randomized trial was to study the applicability of IMT physiotherapy and its specific effects on immediate recovery of pulmonary function as compared to conventional water bottle PEP -physiotherapy in surgical patients after lung resections. We present the following article in accordance with the CONSORT reporting checklist (available at http://dx.doi.org/10.21037/jtd-201668).

\section{Methods}

The study consisted of a total of forty-two patients undergoing major pulmonary resection surgery at Tampere Heart Hospital, Tampere, Finland, from May 2013 to
February 2016. Recruitment ended as intended when predefined number of patients [44] were recruited. Patients with co-operative problems such as psychiatric illness, severe respiratory neurological illness, patients who were under influence of intoxicating agents upon entry into the hospital, infectious lung or other contagious infection (e.g., tuberculosis), or severe respiratory failure $\left(\mathrm{SpO}_{2}<90\right.$ or $\mathrm{pO}_{2}<8$ or respiratory rate at rest over $25 / \mathrm{min}$ or night-time oxygen therapy) were excluded from the study. Patients in special groups or minors were not recruited for this study. One patient declined to participate in the study and two patients were excluded immediately postoperatively when the operation resulted in no major lung parenchyma resection. Participants were enrolled by the investigators (JL) and after signing the informed consent files the patients were randomly allocated to two treatment groups. Randomization was generated by computer-based random allocation with a target of 44 randomized cases to cover potential later patient withdrawals.

Randomization was 1:1 to parallel open label groups of conventional respiratory physiotherapy group (water bottle PEP, 20 patients) and IMT group (IMT, 22 patients). There were no deviations of therapy from the allocated treatment groups nor drop-outs from intended treatment groups. Minor treatment modifications were allowed in case of for example major postoperative air leak. The study protocol (R13037) was conducted in accordance with the Declaration of Helsinki (as revised in 2013) and it was approved by the Ethics Committee of Pirkanmaa Hospital District, Tampere, Finland. Written informed consent was obtained from all the subjects prior to commencing the research. Protocol is filed in ClinicalTrials.gov (Clinical registration number: NCT02931617/U.S. National Library of Medicine, ClinicalTrials.gov).

Chest X-rays (CXRs) were part of the study protocol, and atelectasis, pleural fluid and pulmonary infections were to be evaluated from them. However, study protocol was performed as part of the normal clinical practice in our unit, and routine CXRs from all the patients weren't part of the normal clinical protocol, when the clinicians used other diagnostic tools such as auscultation or bed-side ultrasound to evaluate the chest postoperatively. Preoperatively patients had often thorax CTs instead of CXRs. The same radiologist evaluated the CXRs from all patients, measured and marked the area of the air-containing lung, the areas of atelectasis, the presence and size of pneumothorax, the amount of pleural fluid, and calculated the change in the area of the air-containing lung between preoperative and 
postoperative images. The volume and change of the aircontained lung were evaluated in both PA- and lateral views.

The amount of atelectasis was expressed in three levels $(0=$ no atelectasis, $1=$ atelectasis less than $25 \%$ of lung volume, $2=$ atelectasis $25-50 \%$ of lung volume).

The physiotherapist recorded the occurrence and the amount of post-operative air leak in three levels $(0=$ no air leak, $1=$ low grade air leak (less than $200 \mathrm{~mL}$ ), $2=$ moderate or profuse air leak (air leakage of $200 \mathrm{~mL}$ or more).

\section{Physiotherapeutic intervention methods}

The same physiotherapist (HM) instructed the allocated physiotherapy (water bottle PEP or IMT) to the patients and the exercises were done once a day and were guided by the physiotherapist preoperatively and during the first and the second postoperative day. In addition, independent exercises at least five times a day were encouraged to the patients during preoperative day and first and second postoperative days. In the water bottle PEP group, a basic pressure of $10 \mathrm{H}_{2} \mathrm{Ocm}$ was used, but the pressure was temporarily decreased if necessary. In IMT physiotherapy group, a ThresholdIMT (Philips Respironics, Murrysville, Pennsylvania, United States) device was used. Pressure was defined as $20 \%$ of the patient's preoperative maximum inspiratory power (MIP).

In addition, patients were instructed to walk at least 3-4 times at the first postoperative day and at least five times at the second postoperative day. The patients kept a diary of breathing exercises and walks during the study time. Patients were also instructed routine exercises such as shoulder moving and supporting the surgical wound while coughing.

Volumetric spirometry was done on the preoperative day prior to allocation, and on the first postoperative day and the second postoperative day after the physiotherapy exercise. For 19 patients in water bottle PEP group and 18 in IMT group complete lung function results for all three measuring days were available. Walking distances for first and second postoperative days were recorded for all patients and 0 metre indicated no walking. Primary outcome parameters were lung volumes as measured in the volumetric spirometry, and walking distance was defined as a secondary outcome parameter.

\section{Statistical methods}

Based on a pilot study, the $80 \%$ power to detect a postoperative change of $0.3 \mathrm{~L} / \mathrm{sec}$ in FEV1 (with SD 0.3) with type I error level (5\%) was calculated to need 17 patients recruited per group. Statistical comparisons were made with IBM SPSS Statistics for Windows 24 (IBM Corp, Armonk, New York, United States). The normal distribution of numerical values was tested by ShapiroWilk test. In the case of normal distribution, comparison of the mean values of the variables was done with the $t$-test of independent variables. Differences between the classified variables were tested by cross-tabulation and Pearson's $\chi^{2}$ testing.

Differences between intervention groups in spirometry values and walking distances were analyzed using two-way mixed factors repeated measures analysis as a function of time. To examine the appropriateness of the analysis, the normal distribution and possible outliers were assessed, as well as the homogeneity of variances with the Levene test, covariance equivalence with the Box test, and Mauchly's test of sphericity were performed. Differences were considered statistically significant when $\mathrm{P}<0.05$. Other than primary outcomes were measured as continuous variables or frequencies. There were no changes to trial outcomes after the trial commenced.

\section{Results}

Patient demographics and data are summarized in Table 1. There were no statistically or clinically significant differences between the groups in medical conditions or respiratory diseases, BMI or smoking status. Smoking status information remained unknown for one patient in IMT group. The performed operations included lobectomies, bi-lobectomies, and a single lung removal. The operations were performed both in thoracotomy or VATS (video assisted thoracic surgery). There were no statistical differences between the water bottle PEP and IMT groups in the occurrence of different types of operations.

The preoperative pulmonary function tests indicated weakened function in both groups. Occurrence of severe decrease in respiratory function was slightly higher in the IMT group at baseline, but the difference was not statistically significant. Preoperatively the forced expiratory capacity (FVC\%) was $81 \%$ of the reference population values in the PEP group and $94 \%$ in the IMT group $(\mathrm{P}=0.02)$. Similarly, relative peak inspiratory flow $(\mathrm{PIF} \%)$ was on average $75 \%$ in the PEP group whereas in the IMT group it was significantly higher, $96 \%$ of the reference population values $(\mathrm{P}=0.02)$. Other lung function tests 
Table 1 Main parameters of the study patients in the intervention groups

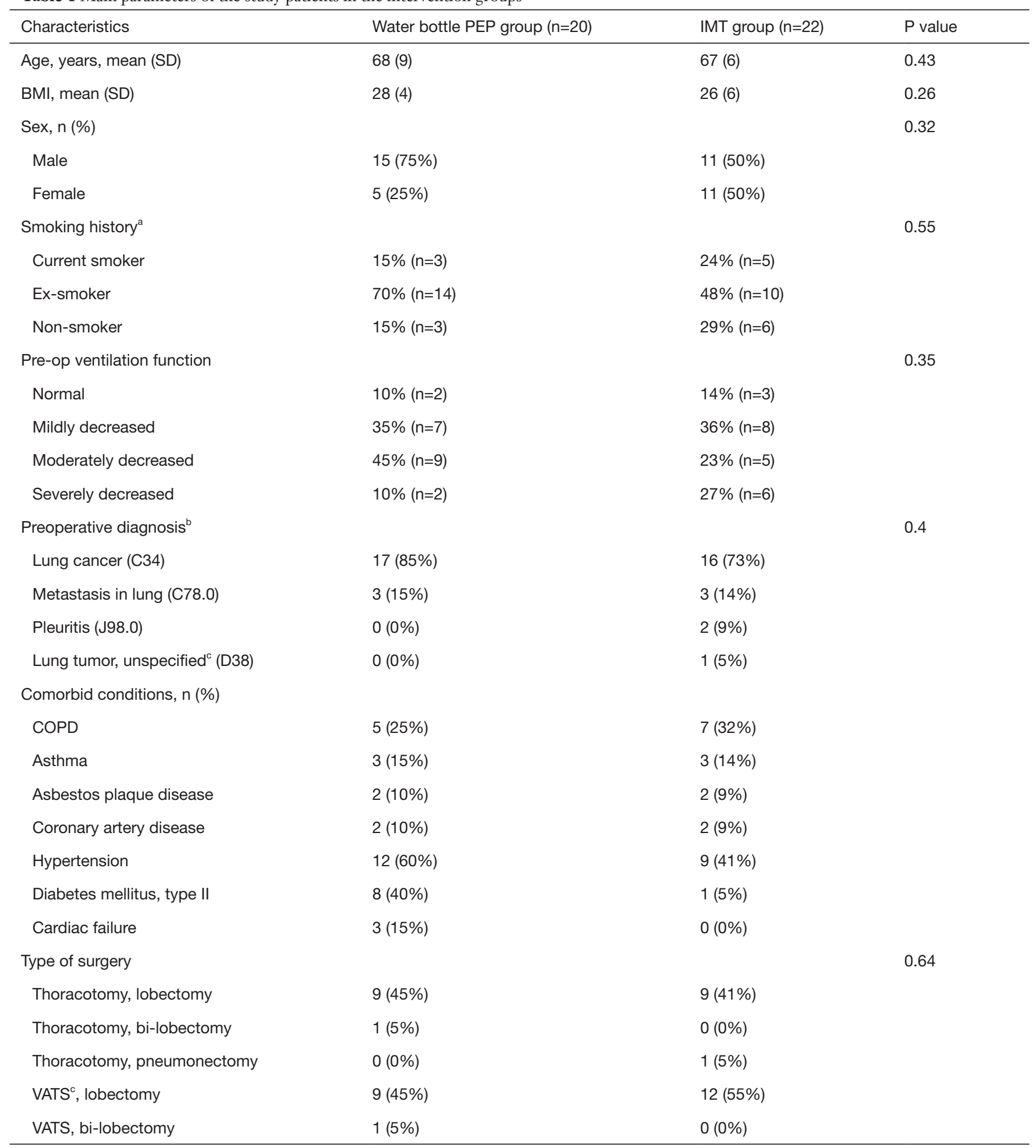

a, current smoker, is smoking at the time of the operation; ex-smoker, quit smoking at least two weeks prior to operation; non-smoker, doesn't have smoking history at all. ${ }^{b}$, with ICD-10 diagnosis codes. ${ }^{c}$, VATS, video assisted thoracic surgery. PEP, positive expiratory physiotherapy; IMT, inspiratory muscle training. 
Table 2 Spirometry values pre- and post-operatively in IMT and water bottle PEP groups

\begin{tabular}{|c|c|c|c|c|c|c|c|}
\hline Variable $^{a}$ & \multicolumn{3}{|c|}{ Water bottle PEP group } & \multicolumn{3}{|c|}{ IMT group } & $P$ value \\
\hline FVC, I/sec (SD) & $3.241(1.12)$ & $2.04(1.07)$ & $2.04(0.65)$ & $3.43(0.83)$ & $2.15(0.73)$ & $2.11(0.62)$ & 0.87 \\
\hline FVC\% (SD) & $80.1(15.6)$ & $50.9(21.5)$ & $51.5(12.3)$ & $95.2(22.7)$ & $58.8(14.4)$ & $57.8(14.1)$ & 0.39 \\
\hline FEV1, I/sec (SD) & $2.27(0.66)$ & $1.49(0.80)$ & $1.45(0.47)$ & $2.42(0.66)$ & $1.57(0.53)$ & $1.54(0.39)$ & 0.90 \\
\hline PEF, I/sec (SD) & $5.92(1.79)$ & $3.69(2.24)$ & $3.63(1.27)$ & $6.30(1.83)$ & $3.88(1.18)$ & $4.01(1.11)$ & 0.92 \\
\hline PEF\% (SD) & $68.8(22.2)$ & $42.0(23.1)$ & $41.9(14.7)$ & $82.4(33.1)$ & $49.7(15.3)$ & $51.6(17.7)$ & 0.70 \\
\hline PIF, I/sec (SD) & $5.22(1.99)$ & $2.89(1.19)$ & $3.25(1.10)$ & $5.64(1.52)$ & $3.50(1.21)$ & $3.71(1.30)$ & \\
\hline PIF\% (SD) & $77.3(21.0)$ & $42.6(12.3)$ & $49.2(14.8)$ & $97.6(30.6)$ & $58.2(16.3)$ & $61.4(17.8)$ & 0.52 \\
\hline Inspiratory force/MIP & $69.8(19.5)$ & & $49.61(13.6)$ & $80.82(23.1)$ & & $58.68(15.7)$ & 0.89 \\
\hline
\end{tabular}

a , forced vital capacity (FVC): the determination of the vital capacity from a maximally forced expiratory effort; FVC\%, forced vital capacity in proportion to control populations results; FEV1, forced expiratory volume during one second; FEV1\%, forced expiratory volume during one second in proportion to control populations results; PEF, peak expiratory flow; PEF\%, peak expiratory flow in proportion to control populations results; PIF, peak inspiratory flow; PIF\%, peak inspiratory flow in proportion to control populations results; FIVC, forced inspiratory vital capacity; FIV1, forced inspiratory volume during one second; Insforce/MIP, inspiratory force/maximal inspiratory power. ${ }^{\text {b }}$, preop pre-operative day. ${ }^{c}$, 1POP first postoperative day. d, 2POP second postoperative day. PEP, positive expiratory physiotherapy; IMT, inspiratory muscle training.

showed no significant differences between the two study groups preoperatively.

The groups were compared according to intention to treat. Mean inspiratory measured force (MIP) was 69.8 $(\mathrm{SD}=19.5) \mathrm{cmH}_{2} \mathrm{O}$ in the water bottle $\mathrm{PEP}$ group and 80.8 $(\mathrm{SD}=23.1) \mathrm{cmH}_{2} \mathrm{O}$ in the IMT group preoperatively. The difference was not statistically significant. At the second postoperative day the mean inspiratory measured force was $49.6(\mathrm{SD}=13.6) \mathrm{cmH}_{2} \mathrm{O}$ in the PEP group and 58.7 (SD $=15.7) \mathrm{cmH}_{2} \mathrm{O}$ in the IMT group. The decrease was similar in the groups and no statistically significant differences between the groups were noticed $(\mathrm{P}=0.07)$.

Detailed parameters of the pulmonary function tests, such as forced expiratory volume in 1 second (FEV1), forced vital capacity (FVC), peak expiratory and inspiratory flow (PEF), forced inspiratory volume in 1 second (FIV1), as primary outcomes were all evaluated using two-way repeated measures analysis of variances at three time points (preoperatively, first and second postoperative day). All pulmonary function values decreased significantly in the first postoperative day compared to preoperative day, but they mostly started to improve at the second postoperative day. The decrease in FVC and FEV1 was similar in both intervention groups and the increase in the second postoperative day was slow. There were no statistically significant differences between groups in FVC and FEV1 (FVC: $\mathrm{P}=0.87$, FEV1: $\mathrm{P}=0.90$ ). In the IMT group the increase in the PEF was slightly higher postoperatively, but the difference was not statistically significant $(\mathrm{P}=0.92)$. Also, the decrease in FIV1 was slightly smaller in the IMT group at first post-operative day, but there were no significant difference between intervention groups $(\mathrm{P}=0.90)$. The results are presented in the Table 2. In summary, no clinically significant differences were detected between the study groups.

Pulmonary function values were also evaluated as relative values (calculated as a ratio of postoperative to preoperative) (Table 3). The results were similarly analyzed at three time points. PEF was returning towards preoperative value slightly faster in the IMT group, but the overall alteration between the groups showed no statistically significant difference $(\mathrm{P}=0.74)$ (Figure 1). PIF drop at first postoperative day in comparison to preoperative value within IMT group was slightly smaller than in water 
Table 3 Postoperative pulmonary function test values (mean ratio, with SD) in proportion to preoperative value in intervention groups

\begin{tabular}{|c|c|c|c|c|c|}
\hline Variable & \multicolumn{2}{|c|}{ Water bottle PEP group } & \multicolumn{2}{|c|}{ IMT group } & $P$ value \\
\hline FVC & $0.66(0.39)$ & $0.65(0.14)$ & $0.64(0.17)$ & $0.63(0.15)$ & 0.90 \\
\hline FEV1 & $0.69(0.41)$ & $0.65(0.15)$ & $0.67(0.19)$ & $0.67(0.18)$ & 0.86 \\
\hline PEF & $0.66(0.49)$ & $0.63(0.17)$ & $0.64(0.16)$ & $0.67(0.20)$ & 0.74 \\
\hline FIVC & $0.62(0.21)$ & $0.65(0.11)$ & $0.64(0.12)$ & $0.64(0.15)$ & 0.86 \\
\hline FIV & $0.58(0.17)$ & $0.62(0.12)$ & $0.62(0.13)$ & $0.63(0.16)$ & 0.78 \\
\hline
\end{tabular}

${ }^{a}$, 1POP/preop first postoperative value in proportion to pre-operative value. ${ }^{b}, 2 \mathrm{POP} /$ preop second postoperative value in proportion to pre-operative value. PEP, positive expiratory physiotherapy; IMT, inspiratory muscle training; FVC, forced vital capacity; FEV1, forced expiratory volume during one second; PEF, peak expiratory flow; PIF, peak inspiratory flow; FIVC, forced inspiratory vital capacity; FIV, forced inspiratory volume.

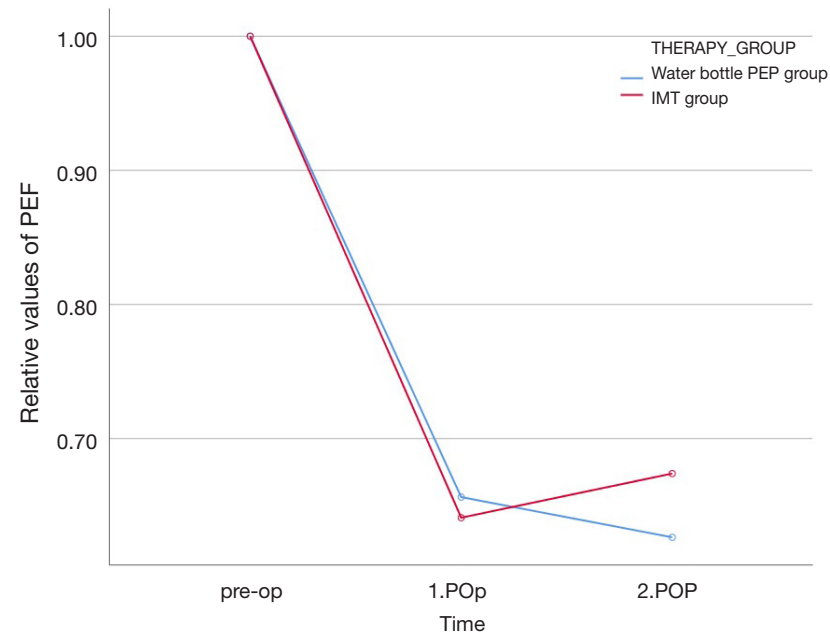

Figure 1 The relative changes of peak expiratory flow at preoperative day, first postoperative day and second postoperative day. Blue line illustrates the water bottle positive expiratory physiotherapy (PEP) group and the red line inspiratory muscle training (IMT) group.

bottle PEP group, but there was no statistically significant alteration between groups $(\mathrm{P}=0.68)$ (Figure 2).

Patients continued independently with the allocated physiotherapy after second postoperative day if they stayed at the ward longer than that. However, pulmonary function values weren't systematically measured after the second postoperative day. Mean hospital stay after operation was 6.6 days ( $\mathrm{SD}=4.1 \mathrm{~d}$ ) in the water bottle PEP group and 5.3 days $(\mathrm{SD}=3.2 \mathrm{~d}$ ) in the IMT group, respectively, but there were no statistically significant difference between intervention groups $(\mathrm{P}=0.29)$.

Walking distance was evaluated as secondary outcome.
The mean walking distance at the first postoperative day in the water bottle PEP group was 312 meters (SD $237 \mathrm{~m}$, range, $8-840 \mathrm{~m}$ ), and in the IMT group 291 meters (SD $225 \mathrm{~m}$, range, $0-800 \mathrm{~m}$ ) respectively. Corresponding values at the second postoperative day were in the water bottle PEP group 463 meters ( $\mathrm{SD}=329 \mathrm{~m}$, range, 20 $1,200 \mathrm{~m})$ and in the IMT group 495 meters $(\mathrm{SD}=331 \mathrm{~m}$, range, $0-1,400 \mathrm{~m}$ ) respectively. The differences in the postoperative walking distances between the groups were not statistically significant $(\mathrm{P}=0.33)$ (Table 4). Pulmonary air leak (mild, moderate or severe) detected with Topaz pleural drainage system was detected in $31 \%$ of patients 


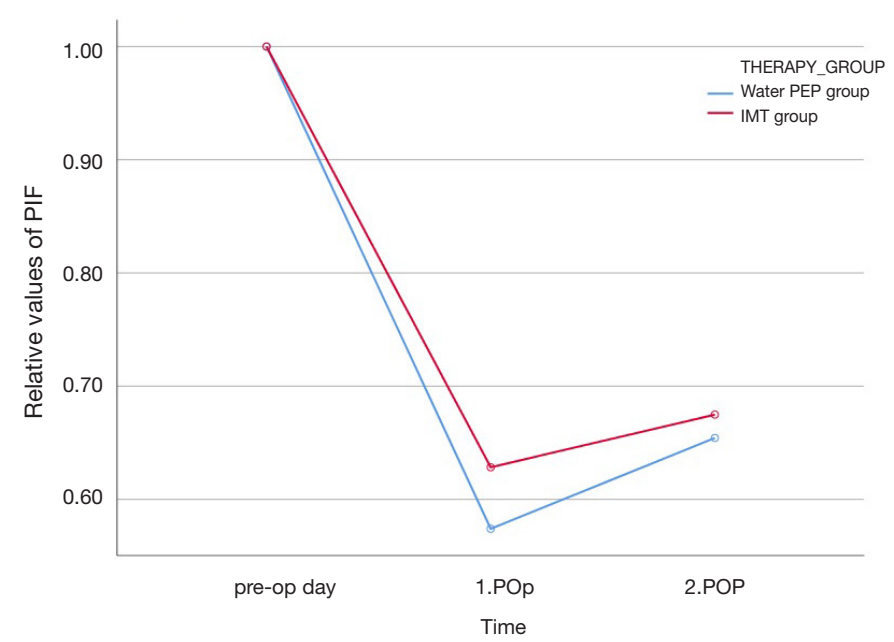

Figure 2 The relative changes of peak inspiratory flow at preoperative day, first postoperative day and second postoperative day. Blue line illustrates the water bottle positive expiratory physiotherapy (PEP) group and the red line inspiratory muscle training (IMT) group.

Table 4 Walking distance within intervention groups at first and second postoperative day

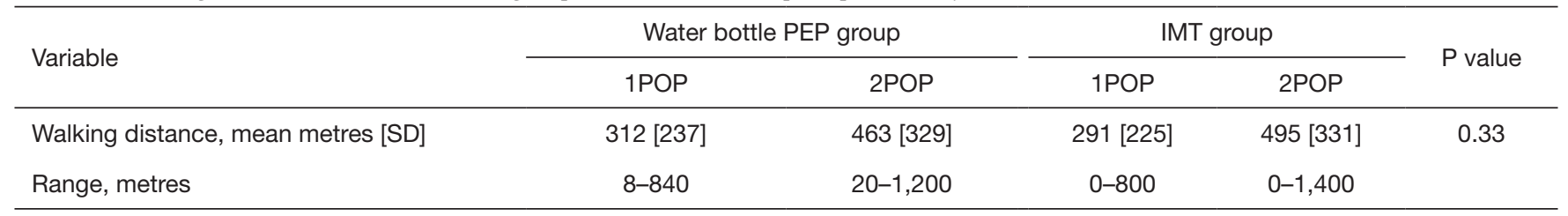

PEP, positive expiratory physiotherapy; IMT, inspiratory muscle training.

Table 5 Incidence of air leak within intervention groups at first and second postoperative day

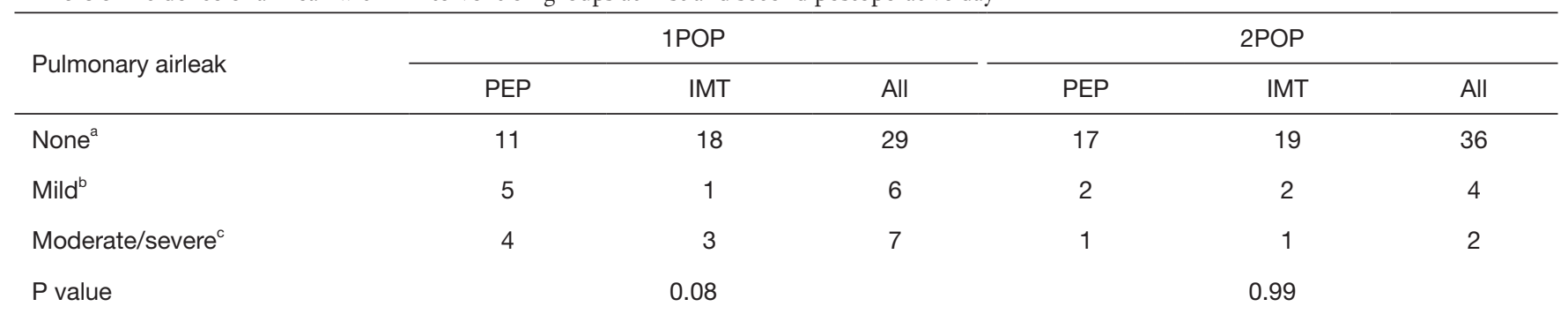

a, none no air leak; ${ }^{b}$, mild air leak less than $200 \mathrm{~mL} ;{ }^{~}$, moderate/severe air leak $200 \mathrm{~mL}$ or more. PEP, positive expiratory physiotherapy; IMT, inspiratory muscle training.

at the first postoperative day and with $14 \%$ of patients at second postoperative day as demonstrated in Table 5. There was no statistically significant difference in occurrence of air leak between intervention groups $(1 \mathrm{POP} \mathrm{P}=0.11 ; 2 \mathrm{POP}$ $\mathrm{P}=0.99)$. Pleural drainage was continued mean 4.1 days after operation in the water bottle PEP group and 3.7 days in the IMT group respectively. There were no statistically significant differences between intervention groups in the length of chest tube drainage time $(\mathrm{P}=0.65)$.

Water resistance with water bottle PEP was decreased to 7 or $5 \mathrm{H}_{2} \mathrm{Ocm}$ if air leak increased during physiotherapy to $200 \mathrm{~mL}$ or more, or if smaller increases were detected at other times, respectively. Water resistance had to be reduced with five water bottle PEP group patients at the first 
Table 6 Deviations in the physiotherapy within intervention groups at first and second postoperative day

\begin{tabular}{|c|c|c|c|c|c|c|}
\hline Implementation of physiotherapy & \multicolumn{3}{|c|}{ 1POP } & \multicolumn{3}{|c|}{ 2POP } \\
\hline No change in physiotherapy ${ }^{a}$ & 14 & 22 & 34 & 17 & 22 & 39 \\
\hline Reduced water pressure ${ }^{b}$ & 5 & 0 & 6 & 2 & 0 & 2 \\
\hline No physiotherapy ${ }^{c}$ & 1 & 0 & 1 & 1 & 0 & 1 \\
\hline
\end{tabular}

${ }^{\mathrm{a}}$, normal physiotherapy, physiotherapy performed as designed. ${ }^{\mathrm{b}}$, reduced water pressure, water pressure reduced to 7.5 or $5 \mathrm{H} 2 \mathrm{Ocm}$ in water PEP physiotherapy due to increased air leak during physiotherapy. ${ }^{c}$, no physiotherapy, physiotherapy halted due to major air leak during physiotherapy. PEP, positive expiratory physiotherapy; IMT, inspiratory muscle training.

postoperative day and two at the second postoperative day because of the air leak. With one patient the water bottle PEP training had to be interrupted at first postoperative day, because of air leak that got worse during PEP physiotherapy. Air leak decreased in one IMT group patient during physiotherapy at both first and second postoperative days. There was a statistically significant difference between intervention groups in physiotherapy feasibility at first postoperative day $(\mathrm{P}=0.01)$ as demonstrated at Table 6 .

Preoperative chest $\mathrm{X}$-rays were available for analysis in 15 patients in water bottle PEP group and in 16 patients from the IMT group. Preoperatively five patients in the water bottle PEP group had atelectasis with less than $25 \%$ of lung volume whereas none of the IMT group patients had atelectasis $(\mathrm{P}=0.012)$. However, no difference in the preoperative ventilated area of the lungs was discovered between the intervention groups.

Air-filled lung area was compared in the chest X-rays (PA- and lateral views) pre- and postoperatively. The full data were achieved in five patients in the water bottle PEP group and in ten patients in the IMT group. The alterations between the treatment groups did not differ statistically significantly, mainly because the resected areas and the contained areas of atelectasis varied widely. No differences in abnormal mucus accumulation, pleural or lung parenchymal infections, or other PPCs were seen between the study groups.

\section{Discussion}

This randomized study showed closely similar short-term results between the established treatments by using water bottle positive expiratory pressure breathing and the novel IMT protocol when pulmonary function tests and walking distance results were compared. Interestingly, we found that patients in the IMT treated group were able to go through physiotherapy during air leak without problems whereas water pressure had to be decreased or physiotherapy stopped with some patients in water bottle PEP treated group. This can favor wider use of IMT physiotherapy.

The evidence of respiratory physiotherapy on patient recovery after thoracic surgery has been contradictory. Some of the studies show no benefit from respiratory physiotherapy, incentive spirometry or IMT physiotherapy after pulmonary surgery (6,27-29). The conclusions drawn from past studies are hampered by varying intervention methods and varying timing and durations of interventions (24). However, some studies have shown that IMT reduces hospitalization time and reduces PPCs (12,21-23).

This study focused on improving patients' immediate respiratory function recovery at the first two postoperative days. The later recovery of the patient's respiratory functions was not monitored because of the short average stay in the hospital. The research setup was done in close accordance with the normal treatment protocol of the unit in order to limit extra costs.

Respiratory function values (FVC, FVC\%, FEV1, FEV1\%, FIVC, FIV1, PEF) decreased significantly on the first postoperative day in comparison to the preoperative day. Respiratory functions were improved somewhat on the second postoperative day, while remaining significantly below preoperative values. The PEF value improved slightly faster in the IMT group than in the water bottle PEP group, but the difference in the improvement was not statistically significant between the groups.

The maximum inspiratory force (MIP) on the second postoperative day was higher in the IMT group than in the water-bottle PEP group, but the difference was not 
statistically significant $(\mathrm{P}=0.07)$. The walking distance in the IMT group improved slightly faster than in the PEP group, but no statistically significant difference between the intervention groups was found. Although there were no statistically significant differences within intervention groups, the water bottle PEP group had somewhat higher number of male patients and lower incidence of COPD, which might increase results of MIP and walking distance in this groups. In contrast, IMT group had one case with pneumonectomy and higher incidence of decreased pulmonary function preoperatively, and this might reduce MIP in this group. However, even with such a short intervention and follow-up period, it was found that IMT physiotherapy was at least as effective as conventional water bottle PEP physiotherapy.

The repeatability of spirometry, especially after surgery, turned out to be a challenge for some patients and, on the other hand, there was lot of dispersion resulting in outlier values in the pulmonary function test results. Pain may be one of the factors that weakens the performance of spirometry, even though the pain was at average well controlled by medication. Patient compliance was good throughout the study thus minimizing the risk of nonparticipation in the self-care and spontaneous training.

Breathing physiotherapy guidance and exercises have been part of our unit's normal postoperative treatment. The study set-up itself did not increase the patients' hospital stay. While evaluating the benefits of physiotherapy, it is important to analyze thoroughly also the cost-effectiveness. Both PEP and IMT physiotherapy are relatively low in costs and do not require expensive equipment, but they require the guidance by a physiotherapist. A more detailed study of cost implications is needed.

The strength of this study was the prospective randomized setting where analyzes were conducted according to the intention to treat principle. The same physiotherapist guided the patient's exercises in both intervention groups, so there should be no difference between the groups in the quality of guidance.

As a weakness of this study may be pointed that the patients or the physiotherapist could not be blinded in relation to the treatment, and this could lead to bias. On the other hand, blinding this type of intervention is technically impossible. Patients' self-guided walking within 24 hours is sensitive to errors due to variations in patient activity and recordings. A six-minute walk under the supervision of a physiotherapist should be a more comparable measure of the differences in exercise ability between patient groups but it is not necessarily a very sensitive test. Also, CXRs were not available from all the patients preoperatively and postoperatively for comparison due to our unit normal clinical practice which utilizes also bed-side ultrasound and auscultation for pleural evaluation.

Postoperative air leaks are fairly common with patients after lung resection surgery, but no difference in air leakage was observed between the intervention groups. The water pressure of water bottle PEP physiotherapy had to be lowered in some patients to control significant air leakage. On the other hand, the IMT physiotherapy was found to reduce air leakage in one case. Difference was observed in the air leak related feasibility of physiotherapy after surgery between intervention groups and this favored IMT at a statistically significant level $(\mathrm{P}=0.01)$.

In future studies on modes of physiotherapy, attention should also be paid to the occurrence and amount of air leak, the feasibility of physiotherapy in cases with air leak, and the possible effects of physiotherapy on air leak. This may be of clinical relevance, especially when selecting modalities of lung rehabilitation after lung resection surgery.

\section{Conclusions}

In conclusion, physiotherapy aiming at improving inspiration seems to be applicable also in patients with resected lung parenchyma and postoperative air leak. No indication of any inferior results was noticed in either treatment modes. We could not demonstrate a presumed difference in ventilation functions or walking distance between the study treatment modes, but important observation was that the inspiratory muscle enhancing treatment is safe and can be particularly useful if the resected lung still leaks air, especially if this is manifested when using positive expiratory pressures.

\section{Acknowledgments}

Funding: This work was supported by Tampere Tuberculosis Foundation and Pirkanmaa Cultural Foundation.

\section{Footnote}

Reporting Checklist: The authors have completed the CONSORT reporting checklist. Available at http://dx.doi. org/10.21037/jtd-20-1668

Data Sharing Statement: Available at http://dx.doi. 
org/10.21037/jtd-20-1668

Conflicts of Interest: All authors have completed the ICMJE uniform disclosure form (available at http://dx.doi. org/10.21037/jtd-20-1668). The authors have no conflicts of interest to declare.

Ethical Statement: The authors are accountable for all aspects of the work in ensuring that questions related to the accuracy or integrity of any part of the work are appropriately investigated and resolved. The study protocol (R13037) was conducted in accordance with the Declaration of Helsinki (as revised in 2013) and it was approved by the Ethics Committee of Pirkanmaa Hospital District, Tampere, Finland. Written informed consent was obtained from all the subjects prior to commencing the research. Protocol is filed in ClinicalTrials.gov (Clinical registration number: NCT02931617/U.S. National Library of Medicine, ClinicalTrials.gov).

Open Access Statement: This is an Open Access article distributed in accordance with the Creative Commons Attribution-NonCommercial-NoDerivs 4.0 International License (CC BY-NC-ND 4.0), which permits the noncommercial replication and distribution of the article with the strict proviso that no changes or edits are made and the original work is properly cited (including links to both the formal publication through the relevant DOI and the license). See: https://creativecommons.org/licenses/by-nc-nd/4.0/.

\section{References}

1. Birim O, Kappetein AP, Bogers AJ. Charlson comorbidity index as a predictor of long-term outcome after surgery for nonsmall cell lung cancer. Eur J Cardiothorac Surg 2005;28:759-62.

2. Falcoz PE, Conti M, Brouchet L, et al. The Thoracic Surgery Scoring System (Thoracoscore): risk model for in-hospital death in 15,183 patients requiring thoracic surgery. J Thorac Cardiovasc Surg 2007;133:325-32.

3. Duggan M, Kavanagh BP. Perioperative modifications of respiratory function. Best Pract Res Clin Anaesthesiol 2010;24:145-55.

4. Ferguson MK, Durkin AE. A comparison of three scoring systems for predicting complications after major lung resection. Eur J Cardiothorac Surg 2003;23:35-42.

5. Hulzebos EH, Helders PJ, Favié NJ, et al. Preoperative intensive inspiratory muscle training to prevent postoperative pulmonary complications in high-risk patients undergoing CABG surgery: a randomized clinical trial. JAMA 2006;296:1851-7.

6. Sharafkhaneh A, Falk JA, Minai OA, et al. Overview of the perioperative management of lung volume reduction surgery patients. Proc Am Thorac Soc 2008;5:438-41.

7. Vagvolgyi A, Rozgonyi Z, Kerti M, et al. Effectiveness of pulmonary rehabilitation and correlations in between functional parameters, extent of thoracic surgery and severity of post-operative complications: randomized clinical trial. J Thorac Dis 2018;10:3519-31.

8. Sanchez-Lorente D, Navarro-Ripoll R, Guzman R, et al. Prehabilitation in thoracic surgery. J Thorac Dis 2018;10:S2593-600.

9. Agostini P, Singh S. Incentive spirometry following thoracic surgery: what should we be doing? Physiotherapy 2009;95:76-82.

10. Weiner P, Man A, Weiner M, et al. The effect of incentive spirometry and inspiratory muscle training on pulmonary function after lung resection. J Thorac Cardiovasc Surg 1997;113:552-7.

11. Kaneda H, Saito Y, Okamoto M, et al. Early postoperative mobilization with walking at 4 hours after lobectomy in lung cancer patients. Gen Thorac Cardiovasc Surg 2007;55:493-8.

12. Zhou K, Su J, Lai Y, et al. Short-term inpatient-based high-intensive pulmonary rehabilitation for lung cancer patients: is it feasible and effective? J Thorac Dis 2017;9:4486-93.

13. Huang J, Lai Y, Zhou X, et al. Short-term high-intensity rehabilitation in radically treated lung cancer: a threearmed randomized controlled trial. J Thorac Dis 2017;9:1919-29.

14. Nici L. Preoperative and postoperative pulmonary rehabilitation in lung cancer patients. Thorac Surg Clin 2008;18:39-43.

15. Steffens D, Beckenkamp PR, Hancock M, et al. Preoperative exercise halves the postoperative complication rate in patients with lung cancer: a systematic review of the effect of exercise on complications, length of stay and quality of life in patients with cancer. Br J Sports Med 2018;52:344.

16. Templeton R, Greenhalgh D. Preoperative rehabilitation for thoracic surgery. Curr Opin Anaesthesiol 2019;32:23-8.

17. Fujimoto $S$, Nakayama T. Effect of combination of preand postoperative pulmonary rehabilitation on onset of postoperative pneumonia: a retrospective cohort study based on data from the diagnosis procedure combination 
database in Japan. Int J Clin Oncol 2019;24:211-21.

18. Freynet A, Falcoz PE. Does non-invasive ventilation associated with chest physiotherapy improve outcome after lung resection? Interact Cardiovasc Thorac Surg 2008;7:1152-4.

19. Varela G, Novoa NM, Agostini P, et al. Chest physiotherapy in lung resection patients: state of the art. Semin Thorac Cardiovasc Surg 2011;23:297-306.

20. Brunelli A, Charloux A, Bolliger CT, et al. ERS/ESTS clinical guidelines on fitness for radical therapy in lung cancer patients (surgery and chemo-radiotherapy). Eur Respir J 2009;34:17-41.

21. Morano MT, Araújo AS, Nascimento FB, et al. Preoperative pulmonary rehabilitation versus chest physical therapy in patients undergoing lung cancer resection: a pilot randomized controlled trial. Arch Phys Med Rehabil 2013;94:53-8.

22. Kendall F, Abreu P, Pinho P, et al. The role of physiotherapy in patients undergoing pulmonary surgery for lung cancer. A literature review. Rev Port Pneumol (2006) 2017;23:343-51.

23. Kendall F, Oliveira J, Peleteiro B, et al. Inspiratory muscle training is effective to reduce postoperative pulmonary complications and length of hospital stay: a systematic

Cite this article as: Lähteenmäki S, Sioris T, Mahrberg H, Rinta-Kiikka I, Laurikka J. Inspiratory training and immediate lung recovery after resective pulmonary surgery: a randomized clinical trial. J Thorac Dis 2020;12(11):6701-6711. doi: 10.21037/ jtd-20-1668 review and meta-analysis. Disabil Rehabil 2018;40:864-82.

24. Ge X, Wang W, Hou L, et al. Inspiratory muscle training is associated with decreased postoperative pulmonary complications: Evidence from randomized trials. J Thorac Cardiovasc Surg 2018;156:1290-300.e5.

25. Pehlivan E, Mutluay F, Balc1 A, et al. The effects of inspiratory muscle training on exercise capacity, dyspnea and respiratory functions in lung transplantation candidates: a randomized controlled trial. Clin Rehabil 2018;32:1328-39.

26. Gosselink R, De Vos J, van den Heuvel SP, et al. Impact of inspiratory muscle training in patients with COPD: what is the evidence? Eur Respir J 2011;37:416-25.

27. Nellessen A, Hernandes NA, Pitta F. Physiotherapy and rehabilitative interventions in patients with chronic respiratory diseases: exercise and non-exercise treatment. Panminerva Med 2013;55:197-209.

28. Ries AL, Bauldoff GS, Carlin BW, et al. Pulmonary Rehabilitation: Joint ACCP/AACVPR Evidence-Based Clinical Practice Guidelines. Chest 2007;131:4S-42S.

29. Freitas ER, Soares BG, Cardoso JR, et al. Incentive spirometry for preventing pulmonary complications after coronary artery bypass graft. Cochrane Database Syst Rev 2012;(9):CD004466. 